\title{
A realidade virtual como instrumento de apoio à conduta fisioterapêutica
}

\author{
The virtual reality as an instrument of support for physiotherapeutic \\ conduct
}

\section{La realidad virtual como instrumento de apoyo a la conducta fisioterapéutica}

\author{
Paulo Henrique Meira DUARTE ${ }^{1}$ \\ Arlete Julian de Souza CÂNDIDO' \\ Rodrigo Cabral ALVES ${ }^{2}$ \\ Jairo Domingos de MORAIS ${ }^{3}$
}

${ }^{1}$ Fisioterapeutas pela Faculdade Maurício de Nassau, UNINASSAU, 58030-000, João Pessoa - PB, Brasil ${ }^{2}$ Graduando de Fisioterapia pela Faculdade Maurício de Nassau, UNINASSAU, 58030-000, João Pessoa - PB, Brasil ${ }^{3}$ Docente Mestre do curso de Fisioterapia da Faculdade Maurício de Nassau, UNINASSAU, 58030-000, João Pessoa - PB, Brasil

\begin{abstract}
Resumo
Introdução: A realidade virtual vem sendo utilizado como uma excelente ferramenta de apoio à prática fisioterapêutica, pois possibilita uma prática eficaz e com redução de riscos à saúde. Objetivo: Analisar a realidade virtual como um potencial instrumento de apoio à prática fisioterapêutica por meio de uma revisão da literatura. Materiais e métodos: O estudo é definido por ser do tipo exploratório através de uma revisão da literatura. Inicialmente, foi elaborado um questionamento para que houvesse um norte na pesquisa, no qual se questionou em quais doenças a realidade virtual vem sendo empregada como conduta terapêutica na realidade da fisioterapia. Resultados: Foi selecionado um total de onze artigos, nos quais passaram por critérios de seleção. Cerca de quatro artigos destinaram-se a investigar a realidade virtual na paralisia cerebral, três artigos com atenção à saúde do idoso e apenas um artigo para a hemiplegia pós-TCE, doenças de Parkinson, hemiparesia crônica pós-AVE e doença de Ménière. Conclusão: A realidade virtual de fato possui inúmeros benefícios e vem sendo utilizada em diversas patologias e condições, no quais obtém resultados significados na rotina terapêutica.
\end{abstract}

Descritores: Terapia de Exposição à Realidade Virtual; Reabilitação; Fisioterapia.

Abstract

Introduction: Virtual reality has been used as an excellent tool to support physiotherapeutic practice, since it allows effective practice and reduction of health risks. Objective: To analyze virtual reality as a potential instrument to support physiotherapeutic practice through a review of the literature. Materials and methods: The study is defined as being of the exploratory type through a review of the literature. Initially, a question was elaborated so that there was a north in the research, in which was questioned in which diseases the virtual reality has been used as therapeutic conduct in the reality of the physiotherapy. Results: A total of eleven articles were selected, in which they went through selection criteria. About four articles were used to investigate the virtual reality in cerebral palsy, three articles with attention to the health of the elderly and only one article for post-TBI hemiplegia, Parkinson's disease, poststroke hemiparesis and Ménière's disease. Conclusion: Virtual reality does indeed have innumerable benefits and has been used in several pathologies and conditions, in which it obtains meaningful results in the therapeutic routine.

Descriptors: Physical Therapy Specialty; Rehabilitation; Physical Therapy Specialty.

\section{Resumen}

Introducción: La realidad virtual viene siendo utilizada como una excelente herramienta de apoyo a la práctica fisioterapéutica, pues posibilita una práctica eficaz y con reducción de riesgos a la salud. Objetivo: Analizar la realidad virtual como un potencial instrumento de apoyo a la práctica fisioterapéutica a través de una revisión de la literatura. Materiales y métodos: El estudio se define por ser del tipo exploratorio a través de una revisión de la literatura. Inicialmente, se elaboró un cuestionamiento para que hubiera un norte en la investigación, en el cual se cuestionó en qué enfermedades la realidad virtual viene siendo empleada como conducta terapéutica en la realidad de la fisioterapia. Resultados: Se seleccionó un total de once artículos, en los que pasaron por criterios de selección. Unos cuatro artículos se destinaron a investigar la realidad virtual en la parálisis cerebral, tres artículos con atención a la salud del anciano y sólo un artículo para la hemiplejia post-TCE, enfermedades de Parkinson, hemiparesia crónica post-AVE y enfermedad de Ménière. Conclusión: La realidad virtual de hecho posee innumerables beneficios y viene siendo utilizada en diversas patologías y condiciones, en el cual obtiene resultados significados en la rutina terapéutica.

Descriptores: Terapia de Exposición Mediante Realidad Virtual; Rehabilitación; Fisioterapia.

\section{INTRODUÇÃO}

A simulação de um ambiente real por meio da comunicação de imagens gráficas e a união de elementos computacionais e ligações sensório-motoras de um indivíduo fomenta o entendimento acerca da realidade virtual (RV). A simulação e a vida real assemelham-se por causa da imersão do indivíduo com a realidade virtual, com isto, ocasiona uma melhor ligação entre o indivíduo e o ambiente virtual ${ }^{1,2}$.

Por questões relacionadas à motivação, por exemplo, a RV vem sendo minuciosamente estudada como um moderno instrumento de apoio à prática fisioterapêutica e podendo ser aplicado de múltiplas formas. Outros benefícios da RV são o controle e aquisição motora e a repetição ${ }^{3-5}$. No processo de reabilitação são importantes vários estímulos no sistema nervoso central, e a RV possui implicações diretas nestes estímulos por meio da interação do indivíduo com ambientes virtuais ${ }^{2}$.

A literatura científica no que se refere à realidade virtual e a sua aplicação na intervenção fisioterapêutica é escassa, necessitando de estudos que investiguem como e em quais patologias a realidade virtual pode ser empregada e quais são os reais benefícios que a RV proporciona ao indivíduo acometido por uma determinada patologia, uma vez que cada patologia possui particularidades.

Portanto, o presente estudo tem por objetivo verificar quais são as patologias que se beneficiam com o uso da realidade virtual como ferramenta de apoio à conduta fisioterapêutica através de uma revisão de literatura.

\section{MATERIAL E MÉTODO}

O presente estudo é caracterizado como exploratório através de uma revisão de literatura. Para alcançar o objetivo almejado foi necessária a formulação de uma pergunta norteadora, no qual foi descrita como: em quais patologias a realidade virtual vem sendo utilizada atualmente e qual a aplicabilidade desta ferramenta tecnológica na intervenção fisioterapêutica?

A coleta de dados iniciou-se entre os meses de junho a agosto do ano de 2017 através da Biblioteca Virtual em Saúde (BVS), no qual foi redirecionada para as bases de dados 
Medical Literature Analysis and Retrieval System Online (MEDLINE) e Literatura Latino-americana e do Caribe em Ciências da Saúde (LILACS).

Os artigos foram encontrados e selecionados por meio do cruzamento dos descritores "realidade virtual" com "fisioterapia" e 'terapia" com "exposição à realidade virtual" na língua portuguesa e "virtual reality" com "physical therapy" e 'therapy" com 'exposure to virtuous reality" na língua inglesa, bem como através da aplicação de critérios de inclusão, tais quais: artigos completos, indexados nas bases de dados supracitadas, no idioma português, publicados entre os anos de 2013 e 2017 e que estivessem em consonância com a pergunta norteadora.

\section{RESULTADOS}

O presente estudo selecionou e analisou um total de 11 (onze) artigos, nos quais obedeceram precisamente todos os critérios anteriormente estabelecidos. No Quadro 1 está descrito as informações pertinentes aos artigos utilizados para a presente revisão.

Desta forma, 11 publicações foram de extrema relevância para a construção desta revisão, no qual resultou em importantes contribuições a temática em questão.

Observou-se na presente revisão que há o predomínio de estudos voltados para analisar a eficácia da realidade virtual aplicada à paralisia cerebral com quatro artigos e em idosos com três artigos, seguido de estudos isolados acerca da hemiplegia pós-TCE, doença de Parkinson, hemiparesia crônica pós-AVE e doença de Ménière.

Quadro 1. Informações relacionadas aos artigos selecionados para a revisão.

\begin{tabular}{|c|c|c|c|c|}
\hline Título & $\begin{array}{c}\text { Autor/ } \\
\text { Ano }\end{array}$ & Objetivos & Resultados & $\begin{array}{l}\text { Base } \\
\text { de } \\
\text { dados }\end{array}$ \\
\hline $\begin{array}{l}\text { Efeito da } \\
\text { Realidade } \\
\text { Virtual no } \\
\text { deslocamento } \\
\text { do COP de } \\
\text { indivíduos com } \\
\text { hemiplegia }\end{array}$ & $\begin{array}{l}\text { Taborda } \\
\text { et al }\end{array}$ & $\begin{array}{l}\text { Avaliar o efeito da } \\
\text { terapia com Wii no } \\
\text { deslocamento do } \\
\text { COP nos sentidos } \\
\text { ântero-posterior e } \\
\text { médio-lateral de } \\
\text { indivíduos } \\
\text { hemiplégicos devido } \\
\text { à sequela de TCE. }\end{array}$ & $\begin{array}{l}\text { Não houve alteração } \\
\text { no deslocamento do } \\
\text { COP nos sentidos } \\
\text { ântero-posterior e } \\
\text { médio-lateral de } \\
\text { pacientes rom } \\
\text { hemiplegia utilizando a } \\
\text { terapia com Wii. }\end{array}$ & LILACS \\
\hline $\begin{array}{l}\text { Realidade virtual } \\
\text { como } \\
\text { possibilidade } \\
\text { terapêutica para } \\
\text { adolescentes } \\
\text { com } \\
\text { encefalopatia } \\
\text { crônica não } \\
\text { progressiva da } \\
\text { infância }\end{array}$ & $\begin{array}{l}\text { Mucelin } \\
\text { et al'. }\end{array}$ & 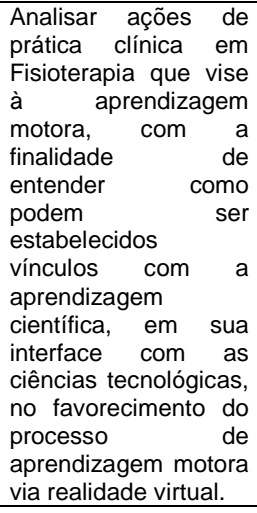 & $\begin{array}{l}\text { Observou-se a } \\
\text { evolução motora dos } \\
\text { sujeitos participantes, } \\
\text { alterando } \\
\text { positivamente o } \\
\text { equilíbrio, a base de } \\
\text { suporte e integração } \\
\text { dos movimentos, } \\
\text { compreendendo uma } \\
\text { possível } \\
\text { potencialização do } \\
\text { aprendizado motor } \\
\text { pela aproximação de } \\
\text { aprendizagem } \\
\text { cientifica. }\end{array}$ & LILACS \\
\hline 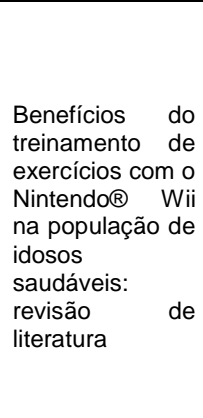 & $\begin{array}{l}\text { Itakussu } \\
\text { et al. }\end{array}$ & $\begin{array}{l}\text { Analisar os benefícios } \\
\text { do treinamento com o } \\
\text { Nintendo Wii para } \\
\text { população de idosos } \\
\text { saudáveis. }\end{array}$ & $\begin{array}{l}\text { O Nintendo® Wii é um } \\
\text { importante instrumento } \\
\text { para prática } \\
\text { fisioterapêutica, com } \\
\text { potenciais benefícios } \\
\text { para os idosos. Devido } \\
\text { às diversas variáveis } \\
\text { encontradas na } \\
\text { metodologia utilizada e } \\
\text { desfechos sugeridos } \\
\text { no estudo, não foi } \\
\text { possível conclusão } \\
\text { definitiva a respeito do } \\
\text { Wii para essa } \\
\text { populacão. essa }\end{array}$ & LILACS \\
\hline $\begin{array}{lr}\text { Treino } & \text { de } \\
\text { equilíbrio } & \text { em } \\
\text { idosos } & \text { com } \\
\text { realidade virtual }\end{array}$ & $\begin{array}{l}\text { Polidoro e } \\
\text { Contencas }^{9}\end{array}$ & $\begin{array}{l}\text { Verificar na literatura } \\
\text { a abordagem de } \\
\text { equilíbrio em idosos } \\
\text { por meio da realidade } \\
\text { virtual. }\end{array}$ & 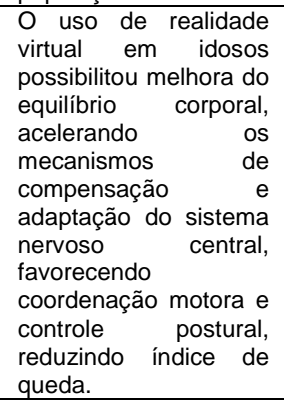 & LILACS \\
\hline $\begin{array}{l}\text { Uso da } \\
\text { realidade virtual } \\
\text { na reabilitação } \\
\text { motora de uma } \\
\text { criança com } \\
\text { paralisia ááxica: } \\
\text { cerebral atál } \\
\text { estudo de caso }\end{array}$ & $\begin{array}{c}\text { Silva e } \\
\text { lwabe- } \\
{\text { Marchese }{ }^{10}}^{\text {and }}\end{array}$ & $\begin{array}{l}\text { Avaliar a influência da } \\
\text { RV com Nintendo Wii } \\
\text { no equilíbrio estático, } \\
\text { dinâmico e marcha de } \\
\text { uma criança com } \\
\text { diagnóstico de } \\
\text { paralisia cerebral } \\
\text { atáxica. }\end{array}$ & $\begin{array}{l}\text { Ficou evidenciado que } \\
\text { o uso do Nintendo Wii } \\
\text { influencia na melhora } \\
\text { do equilíbrio das } \\
\text { crianças quando } \\
\text { complementadas com } \\
\text { o tratamento } \\
\text { cinesioterapêutico, } \\
\end{array}$ & LILACS \\
\hline
\end{tabular}

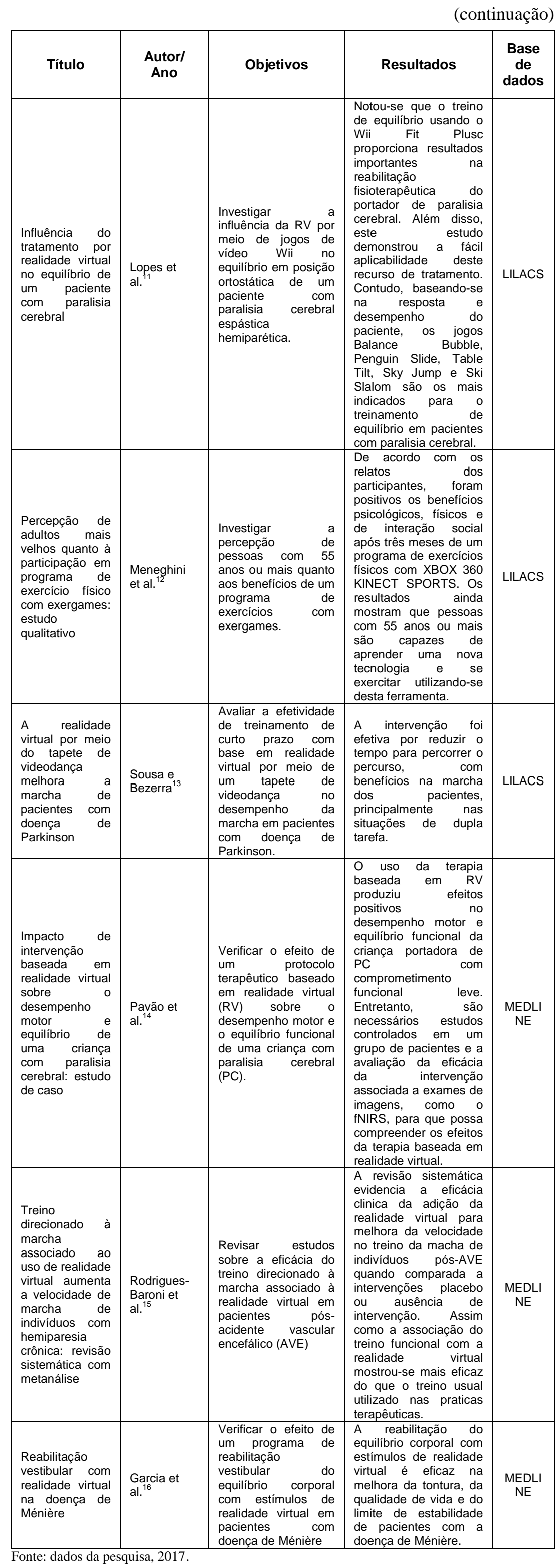

\section{DISCUSSÃO}

Com o avançar dos séculos a tecnologia vem ganhando cada vez mais espaço, novas possibilidades de tratamento ganham força para alavancar condutas fisioterapêuticas, diante disso a RV tem demonstrado a eficácia nos tratamentos. 
A inclusão da RV nos tratamentos em crianças e adultos em diferentes patologias segundo Schiavinato et al. os estudos afirmam que o equilíbrio em pacientes com disfunção cerebelar avaliados após sessões de reabilitação apresenta resultados satisfatórios como no caso de AVE e Doença de Parkinson, onde em seu protocolo a melhora do equilíbrio e da cinemática da marcha são visíveis, portanto os resultados apresentados são encantadores ${ }^{17}$.

Considerando que a funcionalidade engloba todas as funções do corpo e a capacidade do indivíduo de realizar atividades e tarefas, Mucelin, et al. Transmitiu em sua descrição sobre crianças que foram oportunizadas a receber estímulos externos nos primeiros meses sentaram por considerar o aprendizado cognitivo, motor, psicossocial foi fundamental nesse desenvolvimento ${ }^{18}$.

E em idosos a do equilíbrio corpora acelera o mecanicismo de compensação e adaptação do sistema nervoso central reduzindo índices de queda, sendo assim a realidade virtual por meio do tapete de vídeo dança melhora a marcha de pacientes com doença de Parkinson por conter novas maneiras de tratamento ${ }^{19}$.

\section{CONCLUSÃO}

A inclusão da RV nos tratamentos em crianças e adultos em diferentes patologias mostra-se benéfica ao demonstrar melhoras significativas na funcionalidade. As publicações nessa linha de trabalho as abordagens são reduzidas. Fazem-se necessários mais estudos sobre o assunto.

\section{REFERÊNCIAS}

1. Albuquerque EC, Scalabrin EE. O uso do computador em programas de reabilitação neuropsicológica. Psicol Argum. 2007; 25(50):269-75.

2. Treml CJ, Kalil Filho FA, Ciccarino RFL, Wegner RS, Saita CYS, Corrêa AG. O uso da plataforma Balance Board como recurso fisioterápico em idosos. Rev bras geriatr gerontol. 2013; 16(4):759-68.

3. Sharp K, Hewitt J. Dance as an intervention for people with Parkinson's disease: a systematic review and metaanalysis. Neurosci Biobehav Rev. 2014; 47:445-56.

4. Mendes FAS, Arduini L, Botelho A, Cruz MB, SantosCouto-Paz CC, Pompeu SMAA et al. Pacientes com a doença de Parkinson são capazes de melhorar seu desempenho em tarefas virtuais do Xbox Kinect ${ }^{\circledR}$ : uma série de casos. Motricidade. 2015; 11(3):68-80.

5. Bender HV, Jacyntho ZMR, Souza DAP, Borloti E. Medidas comportamentais de presença em ambientes virtuais. CES Psicol. 2016; 9(1):47-64.

6. Taborda B, Briani RV, Silva DO, Pazzinatto MF, Ferreira AS, Segatti $G$ et al. Efeito da Realidade Virtual no deslocamento do COP de indivíduos com hemiplegia. ConScientiae Saúde. 2016; 15(3):354-60.

7. Mucelin M, Carrapatoso BC, Souza PCS, Orsini M. Realidade virtual como possibilidade terapêutica para adolescentes com encefalopatia crônica não progressiva da infância. Rev Bras Neurol. 2015; 51(2):37-44.

8. Itakussu EY, Valenciano PJ, Trelha CS, Marchiori LLM. Benefícios do treinamento de exercícios com o Nintendo(r) Wii na população de idosos saudáveis: revisão de literatura. Rev CEFAC. 2015; 17(3):936-44.

9. Polidoro ACR, Contencas TS. Treino de equilíbrio em idosos com realidade virtual. RBM. 2014; 72(4):153-6.

10. Silva RR, Iwabe-Marchese C. Uso da realidade virtual na reabilitação motora de uma criança com Paralisia Cerebral Atáxica: estudo de caso. Fisioter Pesqui. 2015; 22(1):97-102.

11. Lopes GLB, Yano KM, Tavares NSA, Rego IAO, Marinho RI, Melo LP et al. Influência do tratamento por realidade virtual no equilíbrio de um paciente com paralisia cerebral. Rev Ter Ocup. 2013; 24(2):121-6.

12. Meneghini V, Barbosa AR, Mello ALSF, Bonetti A, Guimarães AV. Percepção de adultos mais velhos quanto à participação em programa de exercício físico com exergames: estudo qualitativo. Ciênc saúde coletiva. 2016; 21(4):1033-41.

13. Sousa ASK, Bezerra PP. A realidade virtual por meio do tapete de videodança melhora a marcha de pacientes com doença de Parkinson. Rev Bras Neurol. 2016; 52(1):21-9.

14. Pavão SL, Arnoni JLB, Oliveira AKC, Rocha NACF. Impacto de intervenção baseada em realidade virtual sobre o desempenho motor e equilíbrio de uma criança com paralisia cerebral: estudo de caso. Rev Paul Pediatr. 2014; 32(4):389-94.

15. Rodrigues-Baroni JM, Nascimento LR, Ada L, TeixeiraSalmela LF. Treino direcionado à marcha associado ao uso de realidade virtual aumenta a velocidade de marcha de indivíduos com hemiparesia crônica: revisão sistemática com metanálise. Braz J Phys Ther. 2014; 18(6):502-12.

16. Garcia AP, Ganança MM, Cusin FS, Tomaz A, Ganança FF, Caovilla HH. Reabilitação vestibular com realidade virtual na doença de Ménière. Braz J Otorhinolaryngol. 2013;79(3):366-74.

17. Monteiro RSJ, Carvalho RJP, Silva EB, Bastos FG. Efeito da reabilitação virtual em diferentes tipos de tratamento. Rev Bras Cienc. 2011; 9(29):56-63.

18. Mucelin M, Cantanhede BC, Souza PCS, Orsini M. Realidade virtual como possibilidade terapêutica para adolescentes com encefalopatia crônica não progressiva da infância. Rev Bras Neurol. 2015; 51(2):37-44.

19. Silva EKR, Macêdo LC. Realidade virtual no treinamento do equilíbrio em idosos: um estudo de revisão. RPF. 2014; 4(2):137-43.

\section{CONFLITO DE INTERESSES}

Os autores declaram não haver conflitos de interesse.

\section{AUTOR PARA CORRESPONDÊNCIA}

\section{Paulo Henrique Meira Duarte}

paulohenriquemd@hotmail.com

Submetido em 15/11/2017 Aceito em 09/01/2018 